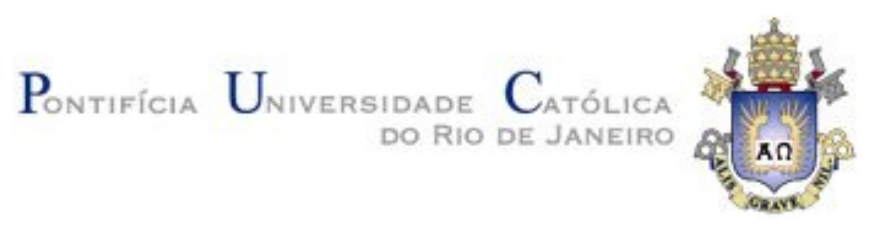

Luana Lachtermacher

O uso de tabelas de decisão para a automação da geração e da execução de casos de teste

Dissertação apresentada como requisito parcial para obtenção do grau de Mestre pelo Programa de Pósgraduação em Informática do Departamento de Informática da PUC-Rio.

Orientador: Prof. Arndt von Staa

Rio de Janeiro, Março de 2010 


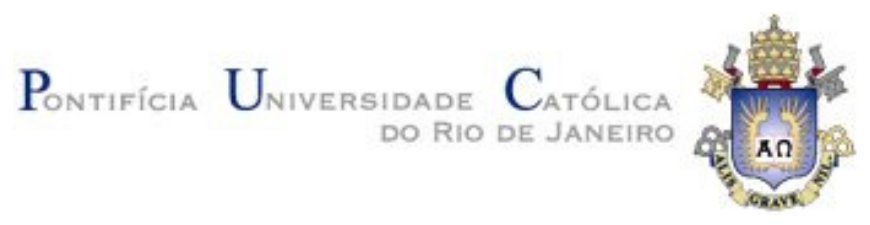

Luana Lachtermacher

\title{
O uso de tabelas de decisão para a automação da geração e da execução de casos de teste
}

Dissertação apresentada como requisito parcial para obtenção do grau de Mestre pelo Programa de Pósgraduação em Informática do Departamento de Informática do Centro Técnico Científico da PUC-Rio. Aprovada pela Comissão Examinadora abaixo assinada.

\author{
Prof. Arndt Von Staa \\ Orientador \\ Departamento de Informática - PUC-Rio \\ Prof. Alessandro Fabrício Garcia \\ Departamento de Informática - PUC-Rio \\ Soeli Fiorini \\ LES - Laboratório de Engenharia de Software - PUC-Rio \\ Prof. José Eugenio Leal \\ Coordenador Setorial do Centro Técnico Científico - \\ PUC-Rio
}


Todos os direitos reservados. É proibida a reprodução total ou parcial do trabalho sem autorização da universidade, do autor e do orientador.

\section{Luana Lachtermacher}

Graduou-se em Bacharel em Informática pela Pontifícia Universidade Católica do Rio de Janeiro em 2007.

Ficha Catalográfica

O uso de tabelas de decisão para a automação da geração e da execução de casos de teste / Luana Lachtermacher ; orientador: Arndt Von Staa - Rio de Janeiro: PUC. Departamento de Informática, 2010.

v., 105 f. : il. ; $29,7 \mathrm{~cm}$

1. Dissertação (mestrado) - Pontifícia Universidade Católica do Rio de Janeiro, Departamento de Informática.

Inclui bibliografia

1. Informática - Teses. 2. Teste de Software. 3. Tabela de Decisão. 4. Geração Automática de Dados. 5. Geração Automática de Scripts. I. Staa, Arndt von. II. Pontifícia Universidade Católica do Rio de Janeiro. Departamento de Informática. IV. Título.

CDD: 004 


\section{Agradecimentos}

Gostaria de agradecer aos meus pais, Gerson e Marly, pelo carinho e amor que sempre meu ajudou a realizar minhas conquistas, ao meu irmão Thiago que sempre era uma fonte de incentivo e ao resto da família pelo carinho e compreensão que tem comigo. Em especial gostaria de agradecer o meu namorado Thuener que nos momentos mais complicados está sempre ao meu lado para conversar e conseguir encontrar o melhor caminho a seguir.

Agradeço ao meu orientador Professor Arndt von Staa pelo seu enorme apoio durante toda a dissertação, sempre conversando e decidindo quais eram os melhores caminhos a seguir.

Aos amigos do Mestrado, que sem eles o mestrado teria sido, com certeza, muito mais complicado.

Agradeço a PUC-Rio e a CAPES pelo apoio financeiro necessário à realização desse trabalho. 


\section{Resumo}

Lachtermacher, Luana; Staa, Arndt Von O uso de tabelas de decisão para a automação da geração e da execução de casos de teste. Rio de Janeiro, 2010. 105p. Dissertação de Mestrado - Departamento de Informática, Pontifícia Universidade Católica do Rio de Janeiro.

Teste é uma etapa muito importante no desenvolvimento de software. No entanto, essa área ainda carece de ferramentas mais eficazes e com um grau de automação maior e mais abrangente do que o atualmente disponível. Uma boa parte das técnicas de geração de casos de teste utiliza explicitamente ou implicitamente tabelas de decisão como instrumento intermediário na geração dos casos de teste específicos. Essa dissertação tem por objetivo desenvolver um processo semi-automático de geração de suítes de teste e que inicia em tabelas de decisão. As suítes geradas devem ser adequadas a ferramentas de execução dos testes totalmente automáticas. Para atingir esse objetivo foram implementados: (i) um editor de tabelas de decisão, (ii) um gerador automático de casos de teste e (iii) um gerador de scripts de teste para o framework FEST. A seguir foram avaliados os benefícios que esse conjunto de ferramentas pode trazer ao processo de automação dos testes, tanto no planejamento (geração de casos de testes valorados a partir dos casos de teste semânticos) quanto na execução dos casos de teste. A avaliação foi baseada em uma série de exemplos envolvendo elementos específicos de interfaces humanas, e, também, na aplicação a um software real.

\section{Palavras-chave}

Teste de Software; Tabelas de Decisão; Geração Automática de Suítes de Teste; Geração Automática de Scripts de Teste; 


\section{Abstract}

Lachtermacher, Luana; Staa, Arndt von Using decision tables to automate the generation and execution of test cases. Rio de Janeiro, 2010. 105p. MSc. Dissertation - Departamento de Informática, Pontifícia Universidade Católica do Rio de Janeiro.

Testing is a very important area in the software development. However, this area needs more effective tools with a higher level of automation with are more comprehensive than the tools available today. A large part of the techniques that do generation of test cases use explicity or implicitly decision tables as an auxiliary tool. This dissertation aims to develop a semi-automatic process that has as outputs tests suites that were generated using decision tables. These suites must be appropriate for the test automation tools. To achieve these goals were implemented: (i) an decision table editor, (ii) a automatic generator case test, and (iii) an automatic test scripts generator of the FEST framework. After it was necessary evaluate the benefits that these tools could bring for the test area, both in the planning (generation of valued test cases from semantic test cases) and in the execution of test cases. The evaluation was based on a series of examples involving specific elements of human interfaces, and also in application to real software.

\section{Keywords}

Software Testing; Decision Table; Automatic Test Suite Generation; Automatic Test Script Generation; 


\section{Sumário}

1 Introdução 13

1.1. Objetivo da Dissertação 15

1.2. Tabela de Decisão 15

1.3. Organização da Dissertação 16

2 Estado da Arte $\quad 18$

2.1. Geração automática de casos de teste 18

2.2. Tabelas de Decisão 20

2.3. Geração automática de dados de teste 22

2.4. Diferenças da Dissertação 24

3 Processo de Teste 25

3.1. Visão Geral do Processo 25

3.2. Criação de Tabela de Decisão 27

3.3. Edição de Tabela de Decisão 27

3.4. Geração Automática de Casos de Teste 28

3.5. Geração Automática de Scripts de Teste 29

3.6. Validação do Processo 30

4 Ferramentas 31

4.1. Editor de Tabela de Decisão 31

4.1.1. Processo do Editor 31

4.1.2. Implementação 44

4.2. Gerador de Casos de Teste 48

4.2.1. Processo do Gerador 48

4.2.2. Implementação 52

4.3. Gerador de Scripts de Teste 53

4.3.1. Processo do Gerador 54

5 Mini Casos $\quad 56$ 
5.1. Campos Numéricos 56

5.1.1. Interface e Especificação 56

5.1.2. Tabela de Decisão 57

5.1.3. Tabela de Decisão Tipada 58

5.1.4. Casos de Teste $\quad 59$

5.1.5. Script Gerado 59

5.2. Campos Alfanuméricos 60

5.2.1. Interface e Especificação 60

5.2.2. Tabela de Decisão 62

5.2.3. Tabela de Decisão Tipada 63

5.2.4. Casos de Teste 64

5.2.5. Script Gerado 65

5.3. Lista 66

5.3.1. Interface e Especificação 66

5.3.2. Tabela de Decisão 67

5.3.3. Tabela de Decisão Tipada 68

5.3.4. Casos de Teste 69

5.3.5. Script Gerado 69

5.4. Combo 70

5.4.1. Interface e Especificação 70

5.4.2. Tabela de Decisão 71

5.4.3. Tabela de Decisão Tipada 72

5.4.4. Casos de Teste 73

5.4.5. Script Gerado 74

5.5. Radio Button 74

5.5.1. Interface e Especificação 74

5.5.2. Tabela de Decisão 76

5.5.3. Tabela de Decisão Tipada 77

5.5.4. Dados gerados 78

5.5.5. Script Gerado 79

5.6. Check box 79

5.6.1. Interface e Especificação 79

5.6.2. Tabela de Decisão 81

5.6.3. Tabela de Decisão Tipada 82 
5.6.4. Dados gerados 83

5.6.5. Script Gerado 84

5.7. Processo 84

6 Estudo de Caso $\quad 86$

6.1. Processo de Teste 86

6.2. Experimento do Teste Manual 94

6.2.1. Primeiro Experimento 94

6.2.2. Segundo Experimento 95

6.2.3. Conclusão dos Experimentos 97

7 Conclusão e Trabalhos Futuros 99

7.1. Contribuições 99

7.2. Trabalhos Futuros 102

8 Bibliografia 104 


\section{Lista de Figuras}

Figura 1: Fases para geração dos testes. ........................................................ 14

Figura 2 - Especificação Z ..................................................................... 23

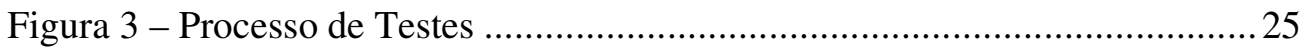

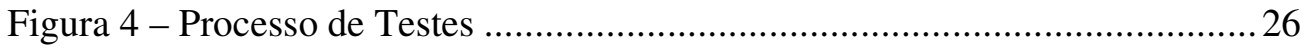

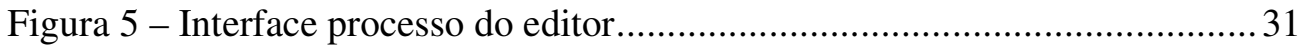

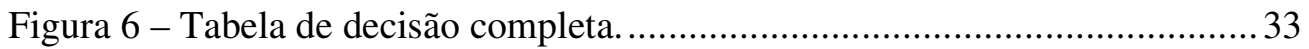

Figura 7 - Interface de inserção de condições especiais...................................... 34

Figura 8 - Interface de inserção de condições com mascaramento...................... 35

Figura 9 - Interface de escolha do tipo de componente...................................... 38

Figura 10 - Interface de definição de uma expressão regular............................. 38

Figura 11 - Arquivo XML de uma tabela de decisão tipada .............................. 42

Figura 12 - Arquivo XML com tabela de decisão com condição com

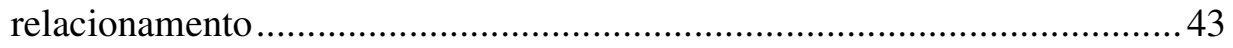

Figura 13 - Tabela de decisão com a definição dos campos .............................. 44

Figura 14 - Diagrama de pacotes do ETD ..................................................... 45

Figura 15 - Diagrama de pacotes do pacote model ............................................. 45

Figura 16 - Diagrama de classes do pacote model........................................... 46

Figura 17 - Diagrama de pacotes do pacote control......................................... 46

Figura 18 - Diagrama de pacotes do pacote view ............................................. 47

Figura 19 - Diagrama de classe de control e interface ....................................... 48

Figura 20 - Processo do Gerador de casos de teste.............................................48

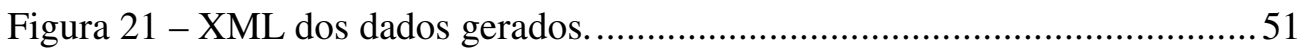

Figura 22 - Diagrama de classe de domínio .................................................52

Figura 23 - Diagrama de classe de control .....................................................53

Figura 24 - Processo do Gerador de scripts de teste ......................................... 54

Figura 25 - Parte inicial do script gerado .................................................... 54

Figura 26 - Script gerado com os casos de teste. .............................................55

Figura 27 - Interface de teste de campos numéricos ........................................56

Figura 28 - Tabela de Decisão do campo numérico...........................................57

Figura 29 - XML com Tabela de Decisão Tipada ...........................................58

Figura 30 - XML com dados do campo numérico .............................................59 
Figura 31 - Parte do script de teste gerado para o exemplo de campos numéricos.

Figura 32- Interface de teste de campos alfanuméricos ...................................6 61

Figura 33 - Tabela de Decisão de campos alfanuméricos .................................62 62

Figura 34 - Arquivo XML com tabela de decisão ...........................................63

Figura 35 - Dados gerados para o campo alfanumérico....................................64

Figura 36 - Script de teste gerado para o campo alfanumérico ..........................65

Figura 37- Interface de teste para componente Lista ......................................... 66

Figura 38 - Tabela de Decisão do componente lista ........................................67

Figura 39- Interface de teste para componente Lista ......................................6 68

Figura 41 - Script de teste gerado para o componente lista..............................69

Figura 42 - Interface de teste para componente combo .................................. 70

Figura 43 - Tabela de Decisão da componente combo ....................................... 71

Figura 44 - Interface de teste para componente combo .................................. 72

Figura 45 - Dados gerados para o componente combo ..................................... 73

Figura 46 - Script de teste do componente combo......................................... 74

Figura 47 - Interface de teste do Radio Button................................................ 75

Figura 48 - Tabela de Decisão do componente Radio Button .............................. 76

Figura 49 - Tabela de Decisão do Button ........................................................... 77

Figura 50 - Dados gerados do Radio Button …….......................................... 78

Figura 51 - Script de teste do Radio Button .................................................. 79

Figura 52- Interface de teste do componente check box .................................. 80

Figura 53 - Tabela de decisão do componente check box ................................ 81

Figura 54 - Tabela de Decisão do Check Box ................................................. 82

Figura 55 - Dados gerados do componente check box....................................... 83

Figura 56 - Script de teste do componente check box...................................... 84

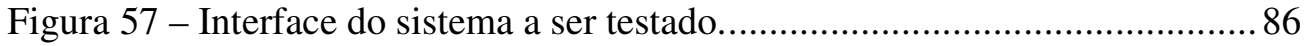

Figura 58 - Tabela de decisão do experimento .................................................. 88

Figura 59 - Arquivo XML com dados do teste ................................................. 90

Figura 60- Casos de teste do script gerado.......................................................91

Figura 61 - Parte inicial do script alterado .................................................... 93

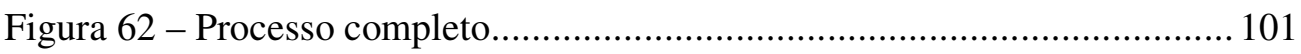

